\title{
Kinematical investigation and regulation of a 4DOF model robot
}

\author{
László Rónai ${ }^{1, *}$, Tamás Szabó ${ }^{2}$ \\ ${ }_{1}^{1}$ Robert Bosch Department of Mechatronics, University of Miskolc, H-3515 Miskolc-Egyetemváros, Hungary \\ ${ }^{2}$ Robert Bosch Department of Mechatronics, University of Miskolc, H-3515 Miskolc-Egyetemváros, Hungary
}

\begin{abstract}
Kinematical investigation and automation of a 4 DOF model robot is discussed in this paper. The well-known Denavit-Hartenberg (DH) parameters are used to prescribe the robot forward-and inverse kinematics. Analytical solution of the inverse kinematical problem is derived by DH homogenous transformation matrix, which gives the relation between the base and the end effector. The article determines the singular positions of the robot. Potential meter and trimmers are attached to the joints of the model robot. A voltage regulator and a controller board of the gripper are designed and built, furthermore an Arduino UNO platform, with a motor shield are used to control the model robot. The main program is written in $C$ code.
\end{abstract}

Keywords: Robot; denavit-Hartenberg parameters; singularity; inverse kinematics; Arduino; microcontroller.

\section{Introduction}

Robots are used for traditional industrial fields [7] and lately for specific purposes [10], [11]. The automation of manufacturing are spreading also in Eastern Europe, mainly due to car industries. The research of robotics and its education also important recently.

The kinematical investigation of a robot is necessary before its practical application. Since there may be some configurations in which the mathematical equations do not provide solutions due to singularities. The singularities of parallel kinematical robots are investigated e.g., in [8] [12].

Application of microcontroller in automation is very popular thanks to its low price. AVR type microcontroller was used to control servo motors of a robot in [9].

This paper contains a case study of a model robot, which can be used for educational purposes. The members of the robot and the motors driving the joints can be purchased commercially [4]. The forward and inverse kinematics of the 4 DOF RRR type robot is examined by DH convention [1], [2]. The 3D model of the robot in in Fig. 1 has been prepared by Creo Parametric 2.0 CAD system.

Section 2 describes the forward and inverse kinematical cases. A simulation program has been developed under a Scilab program system. The singularities of the robot are analyzed in Section 3. The control system of the robot is developed for Arduino UNO platform ([3], [5], [6]), which is based on an AVR type microcontroller. The technical details of the control system are provided in Section 4.

\section{Kinematics of the model robot}

The kinematics of the investigated robot can be described by the help of $\mathrm{DH}$ parameters. The main purpose of the forward kinematics is to determine the 


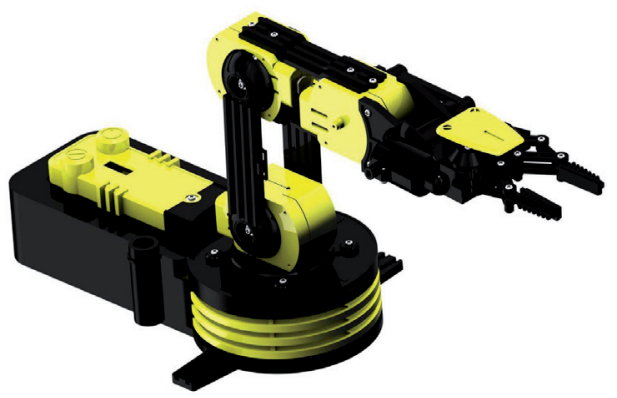

Fig. 1: The 3D model of the robot.

position and orientation of the end effector given the values for the joint variables of the robot [2]. The coordinate systems attached to the joints are shown in Fig. 2. One should give four different parameters $\left(a_{i}, b_{i}, J_{i}, \beta_{i}\right)$ to give the relative homogenous transformations between the joints. The DH paramteres are defined as follows [6]:

- $a_{i}$ is the link length, i.e., the distance of axes $Z_{i-1}$ and $Z_{i}$ (normal transverse, which shouldn't be negative),

- $b_{i}$ is the link offset, i.e., the coordinate $\mathrm{Z}_{\mathrm{i}-1}$ of the section point of axes $X_{i}$ and $Z_{i-1}$,

$\boldsymbol{\square} J_{i}$ is the angle of the joint, i.e., the rotation angle around axis $Z_{i-1}$, which rotates axis $X_{i-1}$ into axis $X_{i}$

- $\beta_{i}$ is the link twist, i.e., the rotation angle around axis $X_{i}$, which rotates axis $Z_{i-1}$ into axis $Z_{i}$.

$\mathrm{D}_{\mathrm{i}-1, \mathrm{i}}=\left[\begin{array}{lll}\cos \left(J_{i}\right) & -\sin \left(J_{i}\right) \cos \left(\beta_{i}\right) & \sin \left(J_{i}\right) \sin \left(\beta_{i}\right) \\ \sin \left(J_{i}\right) & \cos \left(J_{i}\right) \cos \left(\beta_{i}\right) & -\cos \left(J_{i}\right) \sin \left(\beta_{i}\right) \\ 0 & \sin \left(\beta_{i}\right) & \cos \left(\beta_{i}\right) \\ 0 & 0 & 0\end{array}\right.$

The homogenous transformation matrix between the base and the end effector is calculated by the multiplication of the relative homogenous transformation matrices:

$$
\mathrm{DH}_{0,4}=\prod_{\mathrm{i}=1}^{4} \mathrm{D}_{\mathrm{i}-1, \mathrm{i}} \text {. }
$$

The inverse kinematics is the inverse of the forward kinematics. The solution of the problem can be performed analytically, geometrically and numerically. In the analytical approach the first three entries of the last column of the $\mathbf{D H}_{0,3}$ homogenous transformation matrix are utilized to determine the joint angles. One can express the
Due to constructional reason the joint angles fall into the following intervals: $-135^{\circ} \leq J_{1} \leq 135^{\circ}$; $-105^{\circ} \leq J_{2} \leq 105^{\circ} ;-150^{\circ} \leq J_{3} \leq 150^{\circ} ;-50^{\circ} \leq J_{4} \leq 50^{\circ}$. The defined parameters and the coordinate systems are shown in Fig. 2.

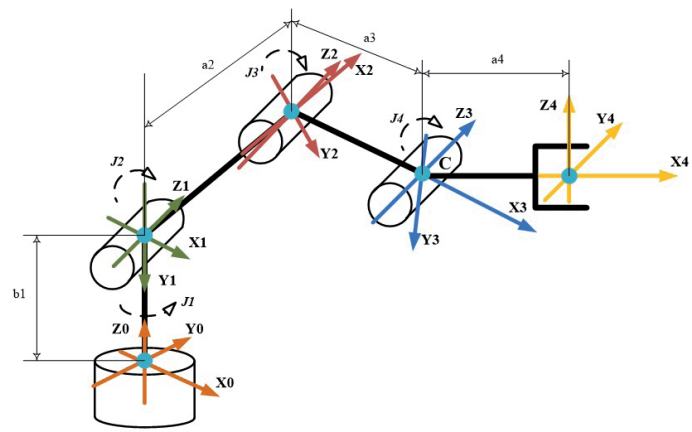

Fig. 2: The defined coordinate systems.

The relative homogenous transformation matrices between the joints are obtained by multiplication of the translational and rotational transformations associated with axes $Z_{i}$ and $X_{i}$ [1]:

$\mathrm{D}_{\mathrm{i}-1, \mathrm{i}}=\mathrm{T}_{\mathrm{zi}} \mathrm{R}_{\mathrm{zi}} \mathrm{T}_{\mathrm{xi}} \mathrm{R}_{\mathrm{xi}}, \quad(\mathrm{i}=1,2,3,4)$.

The investigated robot consist four relative homogenous transformation matrices $\left(\mathbf{D}_{0,1}\right.$, $\left.\mathbf{D}_{1,2}, \mathbf{D}_{2,3}, \mathbf{D}_{3,4}\right)$. A general relative homogenous transformation matrix is given as:

$\left.\begin{array}{l}a_{i} \cos \left(J_{i}\right) \\ a_{i} \sin \left(J_{i}\right) \\ b_{i} \\ 1\end{array}\right]$

coordinates of the 3rd joint denoted by $\mathrm{C}$ in Fig. 2 as follows:

$$
\begin{aligned}
& X_{C}=\cos J 1\left(a_{2} \cos J 2+a_{3} \cos (J 2+J 3)\right), \\
& Y_{C}=\sin J 1\left(a_{2} \cos J 2+a_{3} \cos (J 2+J 3)\right), \\
& Z_{C}-b_{1}=-a_{2} \sin J 2-a_{3} \sin (J 2+J 3) .
\end{aligned}
$$

The angles of the joints $\left(\mathrm{J}_{1}, \mathrm{~J}_{2}, \mathrm{~J}_{3}, \mathrm{~J}_{4}\right)$ can be obtained from equations (4)-(6) after straightforward manipulations. One can determine the rotation angle $\mathrm{J}_{3}$ by calculating cosJ3 and sinJ3: 


$$
\begin{aligned}
& \cos J 3=\frac{X_{C}^{2}+Y_{C}^{2}+\left(Z_{C}-b_{1}\right)^{2}-a_{2}^{2}-a_{3}^{2}}{2 a_{2} a_{3}}, \\
& \sin J 3= \pm \sqrt{1-\cos ^{2} J 3}, \\
& \mathrm{~J}_{3}=\operatorname{atan} 2(\sin (\mathrm{J} 3), \cos (\mathrm{J} 3)),
\end{aligned}
$$

where $\operatorname{atan} 2(\sin (\mathrm{J} 3), \cos (\mathrm{J} 3))$ provides the angular results in the range of $(-\pi \div+\pi)$.

Using trigonometric identities the joint angle $\mathrm{J}_{2}$ also can be determined: $\cos J 2=\frac{\frac{X_{c}}{\cos J 1}\left(-a_{2}-a_{3} \cos J 3\right)+\left(Z_{c}-b_{1}\right) a_{3} \sin J 3}{\left(-a_{2}^{2}-a_{3}^{2}-2 a_{2} a_{3} \cos (J 3)\right)}$
$\sin J 2= \pm \sqrt{1-\cos ^{2} J 2}$

$\mathrm{J}_{2}=\operatorname{atan} 2(\sin (\mathrm{J} 2), \cos (\mathrm{J} 2))$.

The rotation angle of the first joint $\mathrm{J}_{1}$ :

$\mathrm{J}_{1}=\operatorname{atan} 2\left(\mathrm{Y}_{\mathrm{c}}, \mathrm{X}_{\mathrm{c}}\right)$.

The fourth joint angle $\mathrm{J}_{4}$ depends on the practical problem to be treated. A user may define the orientation of the end effector e.g., in always horizontal position, then:

$$
\mathrm{J}_{4}=-\left(\mathrm{J}_{3}+\mathrm{J}_{2}\right) .
$$

A simulation program has been developed under Scilab system by the help (1)-(12) expressions. The robot positions and joint angles can be drawn by the Scilab program. Fig. 3 gives the joint angles and their angular velocities during the motion of the end effector performing an "L" shape path. The geometry of the " $\mathrm{L}$ " shape path is given: the longer part is $0,06 \mathrm{~m}$ and the shorter one is 0,03 $\mathrm{m}$. The translational velocity of the end effector is assumed to be constant and the equidistant steps covered by $d t=0,25 \mathrm{~s}$.

The angular velocities shown in Fig. 3 are calculated by the central difference method:

$\dot{J}_{i}=\frac{J_{i}^{k+1}-J_{i}^{k-1}}{2 d t}$,

where $k$ number of the time step, $\dot{J}_{i}$ the angular velocity of the joint $i$.
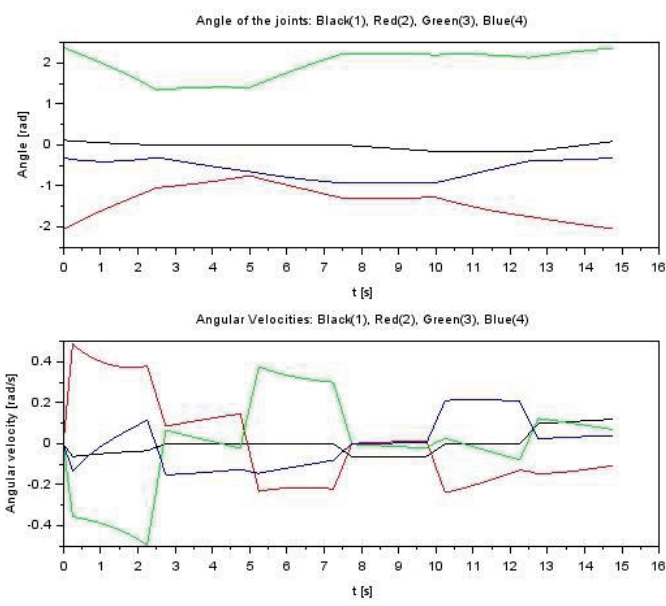

Fig. 3: The angles and the angular velocities of the joints.

\section{Determination of the singularities}

In this Section the singularities of the investigated robot is analyzed. The robot cannot perform arbitrary velocity in a singular position, therefore the inverse kinematical solution in this points provides no solution or infinite number of solutions [1], [2]. The velocity state of the robot in a general position is shown in Fig. 4 using cylindrical coordinate system (CYL).

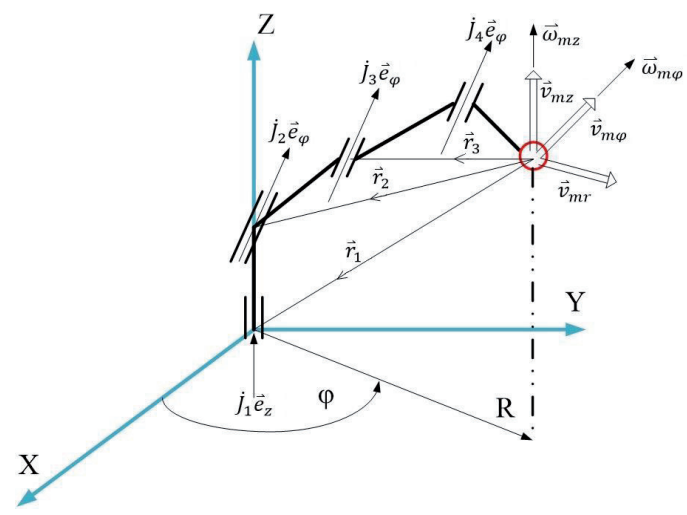

Fig. 4: The velocity status of the end effector.

The position vector $\vec{r}_{\mathrm{i}}$ is expressed in CYL:

$\vec{r}_{\mathrm{i}}=R_{i} \vec{e}_{r}+Z_{i} \vec{e}_{z}$

The velocity and angular velocity of the end effector also given in CYL:

$\vec{v}_{m}=v_{m r} \vec{e}_{r}+v_{m \varphi} \vec{e}_{\varphi}+v_{m z} \vec{e}_{z}$,

$\vec{\omega}_{m}=\omega_{m \varphi} \vec{e}_{\varphi}+\omega_{m z} \vec{e}_{z}$, 
Since the robot system has got 4 DOF, therefore four unknowns can be chosen arbitrarily in (15) and (16). The angular velocity vector of the end effector is equal to the sum of the angular velocities $\dot{J}_{i}$ of the joints. The velocity vector of the end effector is provided by the amount of moments of the angular velocity vectors:

$$
\begin{aligned}
& \vec{\omega}_{m}=\dot{J}_{1} \vec{e}_{z}+\dot{J}_{2} \vec{e}_{\varphi}+\dot{J}_{3} \vec{e}_{\varphi}+\dot{J}_{4} \vec{e}_{\varphi}, \\
& \vec{v}_{m}=\dot{J}_{1} \vec{e}_{z} \times \vec{r}_{1}+\dot{J}_{2} \vec{e}_{\varphi} \times \vec{r}_{2}+\dot{J}_{3} \vec{e}_{\varphi} \times \vec{r}_{3}+\dot{J}_{4} \vec{e}_{\varphi} \times \vec{r}_{4},
\end{aligned}
$$

where the cross products are given in detailed forms:

$$
\begin{aligned}
& \vec{e}_{z} \times \vec{r}_{1}=\vec{e}_{z} \times\left(R_{1} \vec{e}_{r}+Z_{1} \vec{e}_{z}\right)=R_{1} \vec{e}_{\varphi}, \\
& \vec{e}_{\varphi} \times \vec{r}_{\mathrm{j}}=\vec{e}_{\varphi} \times\left(R_{j} \vec{e}_{r}+Z_{j} \vec{e}_{z}\right)=-R_{j} \vec{e}_{z}+Z_{j} \vec{e}_{r}, \\
& (j=2,3,4) .
\end{aligned}
$$

By the help of (17)-(20) a system of linear equations can be written between the velocity state of the end effector and the angular velocities of the joints:

$$
\left[\begin{array}{c}
\omega_{m \varphi} \\
\omega_{m z} \\
v_{m r} \\
v_{m \varphi} \\
v_{m z}
\end{array}\right]=\left[\begin{array}{cccc}
0 & 1 & 1 & 1 \\
1 & 0 & 0 & 0 \\
0 & Z_{2} & Z_{3} & Z_{4} \\
R_{1} & 0 & 0 & 0 \\
0 & -R_{2}-R_{3}-R_{4}
\end{array}\right]\left[\begin{array}{l}
\dot{J}_{1} \\
\dot{J}_{2} \\
\dot{J}_{3} \\
\dot{J}_{4}
\end{array}\right]=\boldsymbol{P} \dot{\boldsymbol{J}}
$$

where $P$ is the matrix of the velocity state and $\underline{\boldsymbol{J}}$ is the vector of the angular velocities:

$$
\boldsymbol{P}=\left[\begin{array}{cccc}
0 & 1 & 1 & 1 \\
1 & 0 & 0 & 0 \\
0 & Z_{2} & Z_{3} & Z_{4} \\
R_{1} & 0 & 0 & 0 \\
0 & -R_{2}-R_{3} & -R_{4}
\end{array}\right], \dot{J}=\left[\begin{array}{c}
\dot{J}_{1} \\
\dot{J}_{2} \\
\dot{J}_{3} \\
\dot{J}_{4}
\end{array}\right] .
$$

Since the robot has got 4 DOF, only 4 parameters can be chosen arbitrarily. Therefore the entries of the vector on the r.h.s. of (21) are not independent, actually $\omega_{m z}$ and $v_{m \varphi}$ can be expressed by each other:

$$
\omega_{m z}=\frac{1}{R_{1}} v_{m \varphi}, \quad \text { if } R_{1} \neq 0 \text {. }
$$

Thus the second equation in (21) can be dropped:

$$
\left[\begin{array}{c}
\omega_{m \varphi} \\
v_{m r} \\
v_{m \varphi} \\
v_{m z}
\end{array}\right]=\left[\begin{array}{cccc}
0 & 1 & 1 & 1 \\
0 & Z_{2} & Z_{3} & Z_{4} \\
R_{1} & 0 & 0 & 0 \\
0 & -R_{2}-R_{3}-R_{4}
\end{array}\right]\left[\begin{array}{c}
\dot{J}_{1} \\
\dot{J}_{2} \\
\dot{J}_{3} \\
\dot{J}_{4}
\end{array}\right]=\boldsymbol{P}_{1} \underline{\dot{J}}
$$

The (24) equation can be expressed in short form:

$\left[\begin{array}{c}\omega_{m \varphi} \\ v_{m r} \\ v_{m \varphi} \\ v_{m \varepsilon}\end{array}\right]=\underline{f_{1}}(\underline{j})$.

The existence of the inverse of $f_{1}$ is examined during the investigation of the singularities of the robot:

$\dot{J}=\underline{f_{1}^{-1}}\left(\omega_{m \varphi}, v_{m r}, v_{m \varphi}, v_{m z}\right)$,

i.e., the rank of matrix $\boldsymbol{P}_{1}$ should be determined. We note that (26) does not exist at $R_{1}=0$, which would lead to ambiguous conclusions in the analysis of the singularity.

If $R_{1}=0$ then the fourth equation in (21) is dropped:

$\left[\begin{array}{c}\omega_{m \varphi} \\ \omega_{m z} \\ v_{m r} \\ v_{m z}\end{array}\right]=\left[\begin{array}{cccc}0 & 1 & 1 & 1 \\ 1 & 0 & 0 & 0 \\ 0 & Z_{2} & Z_{3} & Z_{4} \\ 0 & -R_{2} & -R_{3} & -R_{4}\end{array}\right]\left[\begin{array}{c}\dot{J}_{1} \\ \dot{J}_{2} \\ \dot{J}_{3} \\ \dot{J}_{4}\end{array}\right]=\boldsymbol{P}_{2} \underline{\dot{J}}$.

The existence of the inverse of $\underline{f_{2}}$ now can be examined:

$\dot{J}=\underline{f_{2}^{-1}}\left(\omega_{m \varphi}, \omega_{m z}, v_{m r}, v_{m z}\right)$,

Those configurations of the robot in which the rank of $\boldsymbol{P}_{1}$ or $\boldsymbol{P}_{2}$ is less than 4 are singular. Matrix $\boldsymbol{P}$ is known as the robot Jacobian.

Analyzing the all the possible configurations of the robot taking into consideration the constraints on the angles of the elbows one can conclude that the end effector is situated on a truncated hemisphere with maximum projection distance of the robot (Fig. 5).

The search of the singular positions by $\boldsymbol{P}_{1}$ was completed in oblique configuration of the robot shown in Fig. 6. (a). In vertical configuration of the 


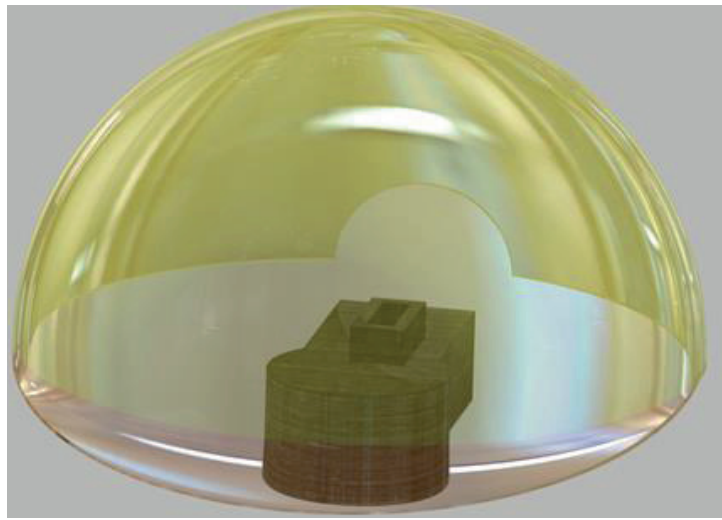

Fig. 5: Singular positions of the end effector.

robot (Fig. 6 (b)) matrix $\boldsymbol{P}_{2}$ was used. In both cases the ranks of the matrices are equal to 3 . In a general oblique configuration all the coordinates of the velocity state $\omega_{m \varphi}, \omega_{m z}, v_{m r}, v_{m \varphi}, v_{m z}$ maybe different from zero, while in vertical configuration both $v_{m \varphi}$ and vmz will be zero.

It is interesting that in special nonsingular configurations shown in Fig. 7 when $R_{1}=0$, the velocity $v_{m \varphi}$ is equal to zero.

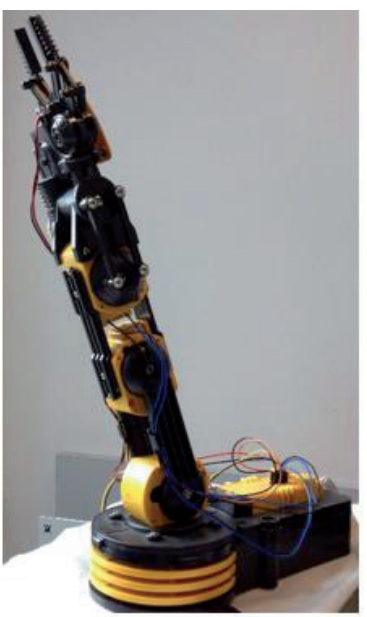

(a)

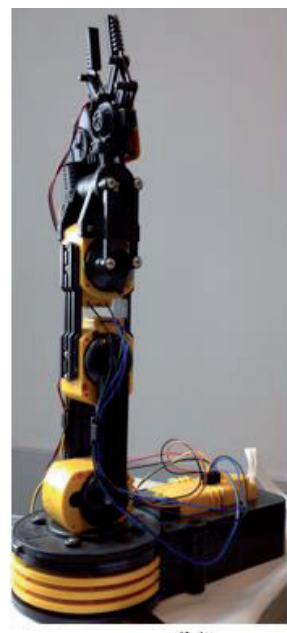

(b)
Fig. 6: The defined singularity states.

\section{Control system of the robot}

The control system of the robot is based on an AVR type microcontroller embedded into an Arduino UNO platform. The system is expandable with different modules, which allowed to attach a motor shield. This shield contains two quadruple half H-bridges (L293D) in order to drive 4 independent DC motors. Furthermore there are

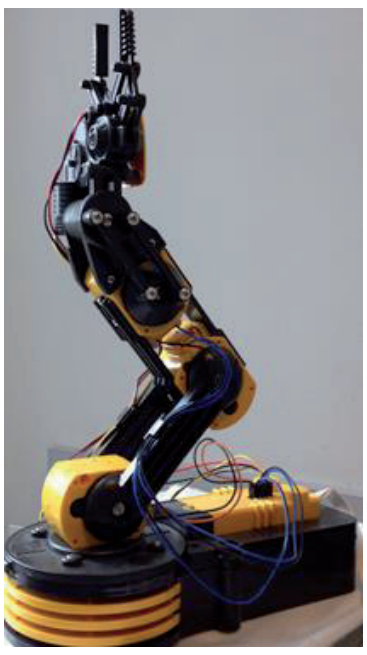

Fig. 7: Nonsingular configuration where $v_{m \varphi}=0$.

another opportunities to control 2 servo motors and one stepper motor. At present the robot works with sequential regulation, the controller program is written in C code.

In addition to the four DC motors driving the joints, which are controlled by Arduino, the gripper of the end effector is driven also by DC motor to which an extra circuit has been developed. The schematic of the developed circuits on the same board are shown in Fig. 8. The part (a) of the board is the voltage regulator and the part (b) is the controller of the fifth DC motor.
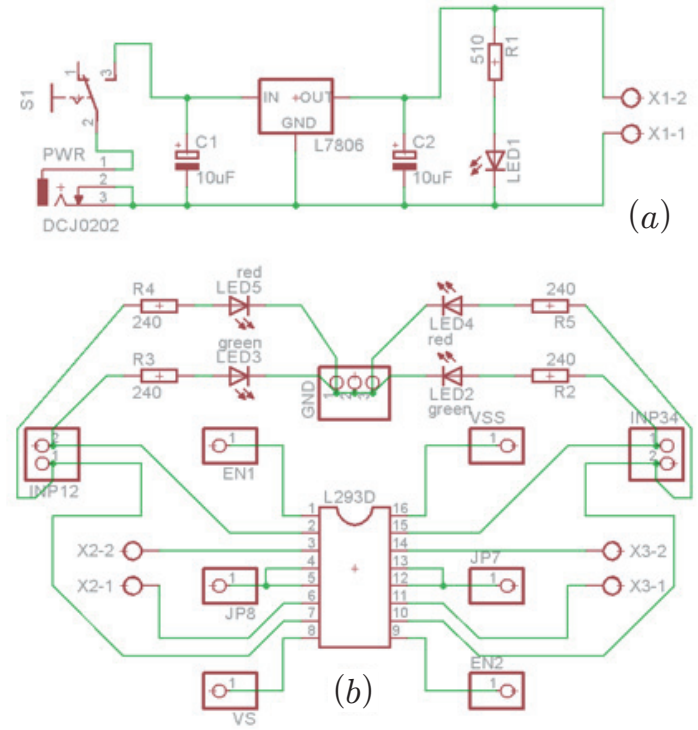

Fig. 8: The schematics of the end effector: (a) voltage regulator (b) DC motor controller. 
The flow chart of the control system is shown in Fig. 9, $\mu C$ denotes the microcontroller.

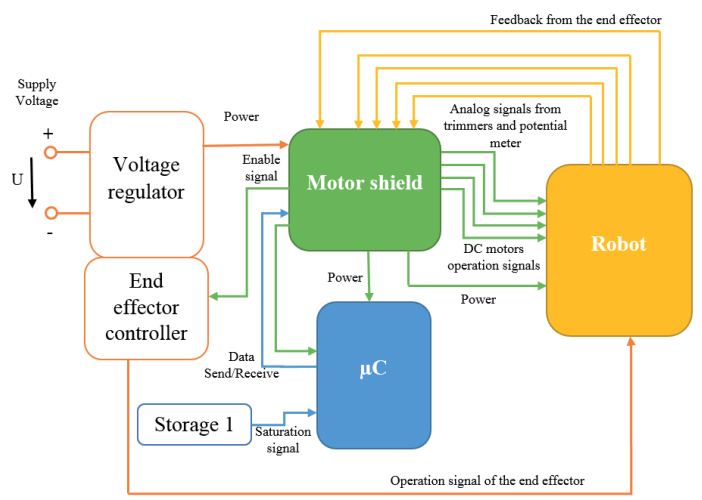

Fig. 9: The flow chart of the operation of the robot.

To make clear the flow chart the operation signals of the four DC motors are given on the right side of the motor shield denoted by green arrows. The analog signals from the three trimmers and one potential meter connected to the motor shield are shown by yellow arrows on the top side of the robot block.

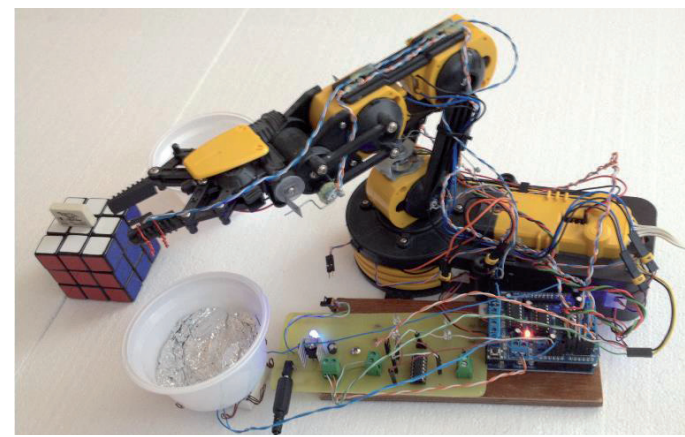

Fig. 10: The controlled robot.

The operation voltage is $6 \mathrm{~V}$, which is provided by an L7806 type voltage regulator IC. Measurements of the angle of the joints are performed by three trimmers and one potential meter. The gripping force of the end effector is bounded by a micro switch attached to the gripper. When the switch is pushed with a certain value of force it provides a logical 1 to the microcontroller, thereafter the DC motors is stopped by the microcontroller. In starting position the micro switch gives logical zero due to a $10 \mathrm{k} \Omega$ pull-down resistor. The model robot regulated by microcontroller is shown in
Fig. 10 during working operation. At present state of the research the robot is gripping rubber objects placed on a Rubic's cube then it is transposing to the storage on the left side of the robot. When this storage is full, the rubber object from the cube are moved to the storage on the right hand side.

\section{Conclusions}

In this paper the kinematics of a 4 DOF model robot is analyzed. The robot is developed for educational and research purposes. The article deals with forward and inverse kinematics of the mechanism of the robot. A simulation program has been developed under Scilab program, which can provide the angles of the joints and also the angular velocities of the joints when constant path velocity of the end effector is prescribed.

The determination of the singular positions was also necessary to perform inverse kinematics. The Jacobian of the robot is given in cylindrical coordinate system. The place of the singular positions of the end effector is a truncated hemisphere. The rank of the Jacobian is equal to 3 in any point of the singular surface.

To control the robot an AVR type microcontroller is used. In this state of the development, the robot complete the necessary motions of the programmed path with sequence control.

\section{Acknowledgments}

This research was carried out in the framework of that Center of Excellence of Mechatronics and Logistics at the University of Miskolc.

\section{References and notes}

[1] Király B. Kinematical and dynamical analysis of industrial robots, Miskolc, 1995. (in Hungarian)

[2] Spong W. M. - Hutchinson S. - Vidyasagar M. Robot Modelling and Control, Wiley, 2006.

[3] Arduino platforms, http://arduino.cc/, 2014.

[4] Velleman ${ }^{\circledast}$ KSR10 Robotic Arm - User Manual, 2011.

[5] Rónai L., Szabó T. Inverse kinematical problem of a 4 DOF model robot, OGÉT Conference, Sibiu, 2014. (in Hungarian)

[6] Rónai L., Szabó T. Controlling of a 4 DOF model robot, ISCAME konferencia, Debrecen 2014.

[7] Kulcsár B. Robottechnika, Typotex 2012.

[8] Nabil S., Moshe S. Singularity Analysis of a Class of Composite Serial In-Parallel Robots, IEEE, 2001.

[9] Goldy K., Saahil G., Shitij K. Design and Operation of Synchronized Robotic Arm, IJRET, 2013. 
[10] Kelemen M., Virgala I., Miková L., Frankovský P.: Experimental Identification of Linear Actuator Properties. In: Acta Mechanica Slovaca. Volume 19, Issue 1, Pages 42-47, ISSN 1335-2393.

[11] Kelemenová, T., Duhoň, F., Puŝkár, M., Kelemen, M., Kuryło, P., Prada, E., Liptak, T.: Influence of Pipe Geometric Deviations on In-Pipe Machine Locomotion. In: Applied Mechanics and Materials. Vol.611(2014), p. 221-226. ISSN 1660-9336.

[12] Amir D., Alon W. Graphical Singularity Analysis of Planar Parallel Manipulators, IEEE, Florida, 2006.

\section{Biographical notes}

László Rónai, is a MSc student at the Robert Bosch Department of Mechatronics in University of Miskolc, Hungary. He was born in Miskolc in 1991. He got BSC degree with honours in mechatronical engineering at the University of Miskolc in 2014. He started the master program in 2014 at the Robert Bosch Department of Mechatronics.

Tamás Szabó, is an Associated Professor at Robert Bosch Department of Mechatronics in University of Miskolc, Hungary. He was born in Tolcsva, Hungary in 1956. He got his mechanical engineering diploma at the Technical University for Heavy Industry in Miskolc, Hungary. He received his PhD degree in 1998 at the University of Miskolc. He is the head of the Robert Bosch Department of Mechatronics at the University of Miskolc. 